\title{
Efectos del tiempo de transporte, espera pre-faena y maduración en novillos sobre indicadores de estrés, calidad instrumental y sensorial de la carne ${ }^{\#}$
}

\author{
Effects of transport, lairage and ageing time on stress indicators and on instrumental \\ and sensory quality of beef from steers \\ M Alende ${ }^{\mathrm{a}}$, G Volpi Lagreca ${ }^{\mathrm{a}}$, AJ Pordomingo ${ }^{\mathrm{a}}$, D Pighín ${ }^{\mathrm{b}, \mathrm{c}}$, G Grigioni ${ }^{\mathrm{b}, \mathrm{c}}$, \\ F Carduza ${ }^{\mathrm{b}}$, A Pazos ${ }^{\mathrm{b}}$, F Babinec ${ }^{\mathrm{a}}$, AM Sancho ${ }^{\mathrm{b}}$ \\ anstituto Nacional de Tecnología Agropecuaria, Estación Experimental Anguil, La Pampa, Argentina. \\ 'Instituto Nacional de Tecnología Agropecuaria, Instituto de Tecnología de los Alimentos, Castelar, Buenos Aires, Argentina. \\ ${ }^{\mathrm{c}}$ Consejo Nacional de Investigaciones Científicas y Técnicas (CONICET), Buenos Aires, Argentina.
}

\begin{abstract}
SUMMARY
We evaluated the effects of two journey times ( $3 \mathrm{~h} v s 8 \mathrm{~h}$ ), two lairage times $(2 \mathrm{~h} v s 14 \mathrm{~h}$ ) and four ageing periods $(4,6,8$ and $15 \mathrm{~d})$ on instrumental and sensory characteristics of Longissimus dorsi from Angus steers $(\mathrm{n}=64)$. Additionally, we evaluated the effect of journey and lairage time on stressrelated metabolites and hormones. The instrumental analysis included final $\mathrm{pH}$, meat colour, Warner Brätzler shear force, cooking losses, water-holding capacity, and those obtained in the texture profile analysis. The sensory parameters evaluated were odour, flavour, initial and final tenderness, juiciness and connective tissue. Journey time had no effect on most of the analysed variables. The longer lairage time led to dehydration in the steers, increasing haematocrit and total plasma protein, decreased glycemia and increased urinary concentration of cortisol. Regarding instrumental quality of beef, longer lairage time increased springiness and chewiness in the texture profile analysis, while decreasing tenderness and juiciness at sensory evaluation. The longer ageing time (15 days) decreased Warner Brätzler shear force and hardness in the texture profile analysis, and increased tenderness and juiciness in the sensory analysis. These results indicate that, under the experimental conditions used in this study, journey times of up to $8 \mathrm{~h}$ did not affect neither physiological indicators of stress nor beef quality, while longer lairage time impaired both physiological indicators of stress and beef quality. Ageing time of 15 days improved tenderness and juiciness of beef.
\end{abstract}

Key words: cattle transport, lairage, beef ageing, beef quality.

\section{RESUMEN}

Se evaluaron los efectos de dos tiempos de viaje (TV, $3 \mathrm{~h} v s 8 \mathrm{~h}$ ), dos tiempos de espera pre-faena (TE, $2 \mathrm{~h} v s 14$ h) y cuatro tiempos de maduración (TM, 4, 6, 8 y 15 días) sobre la calidad instrumental y sensorial del Longissimus dorsi (LD) de novillos Angus ( $\mathrm{n}=64)$. Además, se evaluó el efecto de los dos primeros factores sobre metabolitos y hormonas relacionados con el estrés. Las variables instrumentales analizadas fueron pH final, color, fuerza de corte en cizalla Warner Brätzler, mermas por cocción, capacidad de retención de agua y análisis de perfil de textura. Los parámetros sensoriales evaluados por un panel entrenado fueron olor, flavor, terneza inicial y sostenida, jugosidad y tejido conectivo. El TV no tuvo efecto en casi ninguna de las variables analizadas. Un mayor TE produjo mayor deshidratación, aumentando el hematrocrito y las proteínas plasmáticas totales, descenso de la glucemia y aumento de la concentración urinaria de cortisol. En el análisis instrumental de la carne, aumentó la elasticidad y la masticabilidad en el análisis de perfil de textura, y redujo la terneza y la jugosidad en el panel sensorial. El mayor TM (15 d) produjo descenso en la fuerza de corte en cizalla Warner Brätzler, caída de dureza en el análisis de perfil de textura y aumento de terneza y jugosidad. Los resultados sugieren que, bajo las condiciones de nuestro estudio, TV de hasta $8 \mathrm{~h}$ no tendrían efectos negativos sobre los indicadores de stress y la calidad de carne, mientras que TE más largos tendrían cierto efecto negativo sobre dichas variables. Un TM de 15 días mejoró la terneza y la jugosidad de la carne.

Palabras claves: transporte, espera pre-faena, maduración de la carne, calidad de carne.

\section{INTRODUCCIÓN}

El transporte y las operaciones pre-sacrificio están asociados con una serie de eventos estresantes que afec-

Aceptado: 03.10.2013.

\# Proyecto financiado por el Instituto de Promoción de la Carne Vacuna Argentina (IPCVA).

malende@clemson.edu tan el bienestar animal y pueden afectar la calidad de carne (Ljungberg y col 2007, Maria, 2008, Ferguson y Warner 2008). Por este motivo, las acciones tendientes a mejorar los diferentes eslabones de la cadena logística han recibido una creciente atención en los últimos años. La cadena logística incluye actividades como la carga, el transporte de los animales y la descarga en la planta faenadora, así como operaciones en la línea de faena hasta el enfriamiento de las reses (Ljungberg y col 2007). 
Fisher y col (2009) consideran que los aspectos claves con respecto al bienestar animal durante el transporte pueden agruparse en 3 categorías: (1) el estrés y el miedo asociado a las operaciones de carga, transporte y descarga, (2) el ayuno y la deshidratación como factores metabólicos, y (3) los factores térmicos y físicos que puedan afectar la integridad de los animales. Todos estos aspectos se ven afectados por factores como el tiempo de viaje (Gallo y col 2003, Maria y col 2003, Tadich y col 2005), la densidad de carga (Tarrant y col 1988, Eldridge y Winfield 1988, Gallo y col 2005), la utilización o no de períodos de descanso y abrevaje durante el viaje (Tadich y col 2000), el control periódico de la condición de los animales durante el viaje (Tarrant y Grandin 2000), condiciones de manejo y de rutas (Hoonkavara y col 2005), así como las condiciones de humedad y temperatura ambiente (Miranda-de la Lama y col 2009, Fisher y col 2009). Dentro de estos factores, el tiempo de viaje es una de los determinantes del costo biológico del transporte (Maria 2008). Una evaluación del bienestar animal durante el transporte requiere la utilización de indicadores objetivos y fiables. Para este fin se han utilizados indicadores conductuales (Tarrant y col 1988, Broom 2008), fisiológicos (Parrot y col 1998, Knowles y col 1999), hormonales y metabólicos (Warris y col 1995, Tadich y col 2003, 2005).

Aun cuando la calidad del producto no sea un indicador directo de bienestar, el estrés vinculado con las operaciones pre-sacrificio puede tener efectos negativos en la calidad de carne (Miranda de la Lama 2013). Tanto las catecolaminas como el cortisol afectan el metabolismo muscular de los glúcidos, lo que conduce a un descenso en la concentración muscular de glucógeno, elevando el $\mathrm{pH}$ final de la carne y la calidad de la misma (Warriss y col 1995, Watanabe y col 1996, Immonen y col 2000a). Gallo y col (2003) reportaron un aumento en el pH final de la carne, una caída en la luminosidad y un aumento en la proporción de cortes oscuros con aumentos del tiempo de viaje. El tiempo de viaje también ha mostrado efectos detectables en la calidad sensorial de la carne, afectando la terneza y la apreciación general de la carne por parte de un panel sensorial entrenado (Villarroel y col 2003). La maduración puede modificar la terneza y otros parámetros instrumentales y sensoriales de la carne, lo cual podría atenuar el impacto negativo del estrés pre-faena sobre la calidad de la carne (Bruas-Reignier y Brun-Bellut 1996, Campo y col 1999, Herrera-Méndez y col 2006, Papaleo Mazzucco y col 2010).

El objetivo de este estudio fue determinar los efectos de dos tiempos de viaje ( $3 \mathrm{~h} v s 8 \mathrm{~h}$ ) y dos tiempos de espera pre-faena $(2 \mathrm{~h} v s 14 \mathrm{~h})$ sobre indicadores de estrés en novillos, y de los mismos factores combinados con 4 tiempos de maduración de la carne (4, 6, 8 y 15 d) sobre la calidad instrumental y sensorial del músculo Longissimus dorsi.

\section{MATERIAL Y MÉTODOS}

El estudio se realizó en Anguil ( $36^{\circ} 31^{\prime} \mathrm{S}, 64^{\circ}$ 01' O, 152 m snm), Provincia de La Pampa, en el centro de la
República Argentina, en el mes de Junio de 2009 (invierno). La temperatura media para dicho mes es de $8,3^{\circ} \mathrm{C}$ y la temperatura media anual $15,6^{\circ} \mathrm{C}$. Pertenece a la región semiárida pampeana, con una humedad relativa media mensual para el mes de Junio de 72\% (Casagrande y col 2012).

Se usaron 64 novillos Angus $(435 \pm 20,8 \mathrm{~kg}$ ), a los cuales se asignaron 1 de 4 tratamientos según un diseño factorial 2x2, en el que los factores fueron: i) tiempo de viaje a la planta faenadora (TV, $3 \mathrm{~h} v s 8 \mathrm{~h}$ ) y ii) tiempo de espera pre-faena (TE, $2 \mathrm{~h} v s 14 \mathrm{~h}$ ). Todos los animales fueron criados en la Estación Experimental Agropecuaria Anguil del Instituto Nacional de Tecnología Agropecuaria (INTA), la cual también fue punto de origen del transporte, y se alimentaron en pasturas de avena, siendo suplementados con silaje de sorgo granífero. Cabe destacar que los animales procedían del mismo rodeo y se criaron juntos durante su vida, por lo cual no hubo ingreso de animales ajenos al grupo ni modificaciones que pudieran alterar la escala de dominancia dentro del grupo (Mounier y col 2006, Miranda de la Lama y col 2013).

Se utilizó un camión Mercedes Benz 1620 con chasis y acoplado de dos ejes y ruedas traseras duales, con paredes laterales de madera con espacio suficiente para la ventilación y piso con rejilla antideslizante. La carga del mismo se realizó luego de ayuno de $8 \mathrm{~h}$. Para evitar variaciones debidas al clima, calidad de conducción, diseño del camión y condiciones de la carretera, todos los animales fueron transportados el mismo día, por el mismo conductor, utilizando el mismo camión y siguiendo el mismo recorrido. La figura 1 muestra un cronograma de carga y descarga de los animales. Un primer grupo de 16 animales comenzó el viaje en el chasis del camión saliendo de la estación experimental a las 6:00 h y fue transportado durante 5 h. Luego el camión retornó a la estación experimental y cargó un segundo grupo de 16 animales en el acoplado. Ambos grupos viajaron juntos durante $3 \mathrm{~h}$ hacia la planta faenadora, a la que llegaron a las 14:00 h. De esta manera, el primer grupo fue transportado durante $8 \mathrm{~h}$ y el segundo grupo durante $3 \mathrm{~h}$. Dado que la faena se realizó a la 4:00 $\mathrm{h}$ del día siguiente, ambos grupos de animales tuvieron un TE de $14 \mathrm{~h}$. Luego de la descarga y limpieza del camión, esté retornó a la estación experimental para cargar, a las 18:00 h, un tercer grupo de 16 animales que fue transportado durante $5 \mathrm{~h}$. Luego, a las 23:00 h se cargó el cuarto grupo de 16 animales en el acoplado. Ambos grupos viajaron juntos durante $3 \mathrm{~h}$, llegando a la planta faenadora a las 2:00 $\mathrm{h}$ del día siguiente, donde fueron descargados. Estos dos últimos grupos tuvieron un TE de $2 \mathrm{~h}$, ya que todos los animales se faenaron juntos a las 4:00 h. La densidad de carga del camión fue de $446,25 \mathrm{~kg} / \mathrm{m}^{2}$, lo cual está por encima de lo sugerido por Grandin (1997) y Broom (2008) pero representa una densidad de carga habitual en Argentina. La densidad de carga es cercana a la reportada por Gallo y col (2005) en Chile y Huertas-Canén (2009) en Uruguay, aunque superior a lo reportado por Romero y col (2013) en Colombia. En la planta faenadora, los animales descansa- 


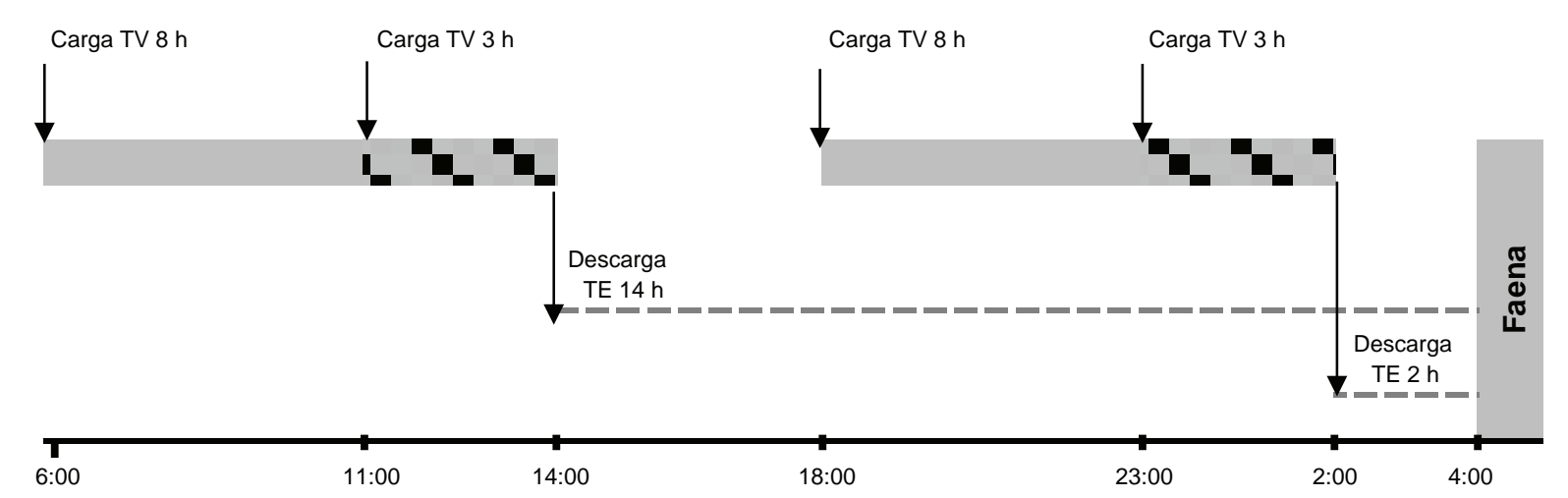

Figura 1. Esquema las labores de carga y descarga de los novillos para llevar a cabo los tratamientos de tiempo de viaje ( $3 \mathrm{~h}$ vs $8 \mathrm{~h}) \mathrm{y}$ tiempo de espera pre-faena ( $2 \mathrm{~h}$ vs $14 \mathrm{~h}$ ).

Scheme of loading and unloading labours of the animals to obtain journey time ( $3 \mathrm{~h} \mathrm{vs} 8 \mathrm{~h}$ ) and lairage time ( $2 \mathrm{~h}$ vs $14 \mathrm{~h})$ treatments.

ron en corrales de espera techados y con piso de concreto antideslizante $\left(2 \mathrm{~m}^{2} /\right.$ animal), con libre acceso a agua.

\section{OBTENCIÓN DE DATOS PRE-FAENA}

Dos días antes de la carga, se tomaron muestras de sangre por venopunción yugular para la determinación del hematocrito basal, el cual fue usado como covariable a la hora de estimar el hematocrito a la faena.

\section{FAENA Y MUESTREO}

El noqueo se realizó con pistola de émbolo cautivo no penetrante. Luego del degüello, se tomaron dos muestras de sangre por animal en tubos estériles comerciales: una con anticoagulante (EDTA) y la otra sin anticoagulante. De la sangre con anticoagulante se tomó una alícuota para calcular el hematocrito. Plasma y suero fueron obtenidos por centrifugación $(2.000 \mathrm{xg}, 10 \mathrm{~min}) \mathrm{y}$ almacenados a $-20{ }^{\circ} \mathrm{C}$ hasta su análisis. Durante el eviscerado, se tomaron muestras de orina de la vejiga urinaria con aguja hipodérmica y jeringa (Lowe y col 2004), las cuales se conservaron a $-80{ }^{\circ} \mathrm{C}$ hasta su análisis. Las canales $(245,5 \pm 25 \mathrm{~kg}$ peso promedio \pm desvío estándar) fueron enviadas inmediatamente a la cámara de frio $\left(4 \pm 2{ }^{\circ} \mathrm{C}\right)$. Dentro de los 45 minutos post sacrificio, se tomaron muestras de Longissimus dorsi para la determinación de glucógeno muscular. Dichas muestras, de $30 \mathrm{~mm}$ de espesor, fueron obtenidas por medio de escalpelo de la porción anterior del músculo, a la altura de la $12^{\circ}$ costilla, de cada hemicanal izquierda. Las muestras fueron comprimidas para eliminar la sangre, colocadas individualmente en recipientes rotulados, sumergidas en nitrógeno líquido y mantenidas a $-80{ }^{\circ} \mathrm{C}$ hasta su análisis.

A las $24 \mathrm{~h}$ post-faena, se midió el $\mathrm{pH}$ final en el músculo Longissimus dorsi de la hemicanal izquierda, por medio de un pH-metro con electrodo de penetración Testo 205
(Testo, Hampshire, Reino Unido). La temperatura al momento de la medición era de $4 \pm 2{ }^{\circ} \mathrm{C}$. Luego, de ambas hemicanales, se cortó con sierra eléctrica una sección del Longissimus dorsi $(\mathrm{n}=128)$ con hueso comprendida entre la $10^{\circ}$ y la $13^{\circ}$ costilla. Todas las muestras (sección completa de músculo con hueso) fueron individualmente identificadas y envasadas al vacío (envasadora Rapivac Optima 800 CL, Argentina; bolsas Cryovac ${ }^{\circledR}$ Barrier Bag 2800 espesor $58 \pm 10 \mu$, Sealed Air Argentina SA, presión de vacío: 28 pulg $\mathrm{Hg}$ ). Para cada músculo, las muestras fueron asignadas al azar a 1 de $4 \mathrm{TM}(4,6,8$ y $15 \mathrm{~d})$. Las muestras fueron maduradas a $2^{\circ} \pm 1^{\circ} \mathrm{C}$ durante el TM asignado, y luego fueron congeladas a $-20^{\circ} \mathrm{C}$ hasta su posterior análisis.

\section{VARIABLES FISIOLÓGICAS Y GLUCÓGENO MUSCULAR}

Las muestras de suero y orina fueron remitidas congeladas al Instituto de Tecnología de los Alimentos de INTA Castelar, Buenos Aires, Argentina, donde se realizaron los siguientes análisis:

Proteínas plasmáticas totales. La concentración de proteínas plasmáticas totales se determinó sobre muestras de suero por medio de la técnica de Bradford (1976), utilizando seroalbúmina bovina (SIGMA, EEUU) como standard.

Glucemia y concentración plasmática de lactato. La glucemia se cuantificó espectrofotométricamente en plasma mediante método enzimático (Trinder 1969). El lactato plasmático se midió con método enzimático comercial (Randox Lab Ltd, Reino Unido).

Concentración urinaria de adrenalina, cortisol y creatinina. Para adrenalina y cortisol urinarios se usó el test de ELISA (BioSource, Bélgica). La creatinina urinaria se determinó mediante kit enzimático para método cinético (Wiener, Argentina). Los niveles de adrenalina y cortisol urinarios se expresaron en $\mathrm{ng} / \mu \mathrm{g}$ de creatinina urinaria. 
Concentración de glucógeno muscular. Se aplicó hidrólisis ácida con $\mathrm{HCl}$ y luego se midieron las unidades de glucosa liberadas mediante el método de Trinder (1969), expresándose como unidades de glucosa-glucógeno/g tejido húmedo (Passonneau y Lauderdale 1974).

\section{ANÁLISIS INSTRUMENTAL DE LA CARNE}

Color. Las muestras fueron descongeladas $24 \mathrm{~h} \mathrm{a} 4{ }^{\circ} \mathrm{C} \mathrm{y}$ luego expuestas al aire 45 min para lograr un adecuado desarrollo del color, el cual se midió con espectrofotómetro de reflectancia BYK-Gardner Espectro-glide 45/0 color gloss (model 9000, EEUU). Se utilizó la escala CIELab , determinándose los parámetros L* (luminosidad; 0 negro, 100 blanco), a* (verde-/rojo+) y b* (azul-/ amarillo+).

Capacidad de retención de agua (CRA). Una sub-muestra $(0,3 \mathrm{~g})$ de cada muestra fue colocada sobre papel de filtro reticulado (grado 1003, Munktell) y comprimida entre dos placas de metacrilato durante $5 \mathrm{~min}$. Por efecto de la presión se liberaron jugos, definiéndose sobre el papel de filtro dos áreas: una central, correspondiente al sector ocupado por la carne, y un anillo perteneciente a la superficie ocupada por el jugo fuera de la carne, y cuya magnitud es inversamente proporcional a la CRA de la carne. El resultado se expresa como el cociente entre las dos superficies.

Fuerza de corte en cizalla Warner-Brätzler (WB) y mermas por cocción. Cada muestra fue pesada y cocida en plancha eléctrica hasta una temperatura interna de $71{ }^{\circ} \mathrm{C}$ (AMSA 1996). Luego fue pesada nuevamente para calcular mermas por cocción y enfriada en heladera a $10 \pm 2{ }^{\circ} \mathrm{C}$ por $24 \mathrm{~h}$. Para la determinación de WB, de cada muestra se extrajeron con sacabocado 8 sub-muestras cilíndrica de $1,25 \mathrm{~cm}$ de diámetro en sentido paralelo a la orientación de las fibras musculares, los cuales fueron cortados utilizando una Cizalla de Warner Bratzler (model 3000; G-T Manufacturing Co., Manhattan, Kansas, EEUU). Los resultados se expresaron en Newton $(\mathrm{N})$.

Perfil de textura. Se usó un texturómetro TA.TX plus (Stable Micro Systems) según las siguientes especificaciones: compresión hasta el 70\%, $1 \mathrm{~s}$ de tiempo de espera entre compresiones sucesivas, celda de carga de $50 \mathrm{~kg}$, velocidad de test de $1 \mathrm{~mm} \cdot \mathrm{s}^{-1}$, velocidad de pre y post test de $3 \mathrm{~mm} \cdot \mathrm{s}^{-1}$, probeta cilíndrica SMS P/35, compresión sobre un eje paralelo a las fibras musculares. La muestra fue cocida en plancha eléctrica de doble contacto hasta una temperatura interna de $71^{\circ} \mathrm{C}$ y luego fue conservada a $5^{\circ} \mathrm{C}$ por $24 \mathrm{~h}$ (AMSA 1996). Por cada muestra se extrajeron con sacabocado entre 6 y 8 sub-muestras cilíndricas de $1,25 \mathrm{~cm}$ de diámetro y $1,25 \mathrm{~cm}$ de alto. Se evaluaron los siguientes parámetros mecánicos, según lo descripto en Caine y col (2003), los cuales se asocian a características de la muestra: dureza 1 (fuerza necesaria para alcanzar un determinado grado de deformación del cubo de carne durante el primer ciclo de compresión, representa la du- reza de la muestra durante la primera mordida, expresado en $\mathrm{N}$ ), dureza 2 (fuerza necesaria para alcanzar un determinado grado de deformación del cubo de carne durante un segundo ciclo de compresión, representa la dureza de la muestra durante la segunda mordida, expresado en N), elasticidad (capacidad de un material deformado de retornar a su condición inicial, es un cociente a partir de la curva fuerza-tiempo), cohesividad (se relaciona con los enlaces internos que dan cuerpo al alimento, es un cociente a partir de la curva fuerza-tiempo) y masticabilidad (dureza x cohesividad x elasticidad, en $\mathrm{N}$ ).

\section{ANÁLISIS SENSORIAL DE LA CARNE}

Se realizó un análisis descriptivo cuantitativo. De cada muestra se tomaron porciones de $2,5 \mathrm{~cm}$ de espesor que fueron descongeladas en heladera $\left(5^{\circ} \mathrm{C}, 48 \mathrm{~h}\right)$ y posteriormente cocidas durante $10 \mathrm{~min}$ en plancha de doble contacto hasta una temperatura interna de $71{ }^{\circ} \mathrm{C}$ (AMSA 1996). Luego se cortaron en cubos de $1 \mathrm{~cm}^{3}$ y fueron servidas a un panel de 8 jueces entrenados, quienes evaluaron dos cubos aleatorios de cada muestra (AMSA 1996). Se usó una escala de 9 puntos para los atributos flavor (combinación característica de gusto, aroma y sensaciones trigeminales), olor, terneza inicial (corresponde a las 3 primeras masticaciones molares de la muestra), terneza sostenida (corresponde a las sucesivas masticaciones), jugosidad y cantidad de tejido conectivo (relaciona el tamaño de la muestra al inicio y luego de la masticación). La escala de 9 puntos cubrió un rango desde $1=$ extremadamente suave (olor y flavor), extremadamente duro (terneza inicial y final), extremadamente seco (para jugosidad) y nula percepción de tejido conectivo a $9=$ extremadamente intenso (flavor y olor), extremadamente tierno (terneza inicual y sostenida), extremadamente jugoso (jugosidad) y mucha percepción de tejido conectivo.

\section{ANÁLISIS ESTADÍSTICO}

Los datos se analizaron utilizando el Proc Mixed de SAS (SAS, 1990). Para el caso de las variables sanguíneas, urinarias y glucógeno muscular, se analizó como un diseño factorial utilizando un modelo incluyendo el efecto de TV, TE y la interacción entre los factores. Para el caso de hematocrito, se utilizó el hematocrito basal obtenido previo al transporte de los animales como covariable. Cuando el efecto de tratamientos fue significativo $(\mathrm{P}<0,05)$ las medias fueron comparadas mediante test de Tukey. Para el análisis de las variables de calidad instrumental de la carne, se trató de un diseño en bloques incompletos al azar, con el animal como criterio de bloqueo y una asignación de tratamientos según diseño factorial 2x 2 × 4 (TV, TE y TM). En el caso del análisis sensorial, el modelo incluyó los tres factores (TV, TE, TM), sus interacciones, el animal como criterio de bloqueo, más el efecto del panelista y de la sesión de evaluación. 


\section{RESULTADOS}

Dado que no existieron interacciones significativas entre los factores, los resultados se presentan mostrando los efectos de los factores principales.

\section{VARIABLES SANGUIINEAS Y URINARIAS Y GLUCÓGENO MUSCULAR}

No hubo efectos significativos del TV sobre el hematocrito ni sobre las proteínas plasmáticas totales. En cambio, sí hubo un efecto significativo del TE para ambas variables. Con el TE más prolongado, tanto el hematocrito como la concentración de proteínas plasmáticas totales se incrementaron (cuadro 1). El TV no afectó ninguno de los parámetros relacionados con el metabolismo glucídico (glucemia, glucógeno muscular y lactato plasmático). En cambio, se observó un descenso de la glucemia con el TE más prolongado (cuadro 1). Los animales sometidos al mayor TE tuvieron mayores niveles de cortisol urinario mientras que los animales con el menor TE tuvieron mayores niveles de adrenalina urinaria, mientras que el TV no afectó el nivel de hormonas urinarias (cuadro 1).

\section{ANÁLISIS INSTRUMENTAL DE LA CARNE}

$p H$ final. No hubo efecto del TV ni del TE sobre el $\mathrm{pH}$ final de la carne (cuadro 2). El pH final promedio fue 5,51 y no se registraron valores de $\mathrm{pH}$ final por encima de 6,00 .

Color de músculo. No se registraron efectos significativos del TV ni del TE. En cambio, el TM afectó los valores del parámetro a* (cuadro 2), el cual aumentó del día 4 al día 6, descendió al día 8 y volvió a aumentar al día 15 .

Fuerza de corte en cizalla de Warner-Brätzler y mermas por cocción. Ninguna de estas dos variables mostró efecto de TV o TE (cuadro 2). El TM afectó tanto a WB como a las mermas por cocción. La WB resultó significativamente menor al día 15 de TM (cuadro 2). Las mermas por cocción fueron más bajas al día 4 y 8 de TM, mostrando valores más altos al día 6 del TM y valores intermedios al día 15, por lo que el comportamiento fue irregular.

Capacidad de retención de agua. La CRA no fue afectada por ninguno de los factores considerados (cuadro 2)

Perfil de textura. El cuadro 2 muestra los resultados obtenidos en el análisis del perfil de textura para Longissimus dorsi. Como puede observarse, no se observó efecto de TV, pero si hubo efecto de TE en elasticidad y masticabilidad, las cuales mostraron valores más altos con el mayor TE. Además, el TM afectó a dureza 1 y dureza 2, las cuales mostraron un significativo descenso en el TM de 15 d. Llamativamente, la elasticidad apareció aumentada en el TM de $15 \mathrm{~d}$.

\section{ANÁLISIS SENSORIAL DE LA CARNE}

El mayor TE redujo significativamente la terneza inicial, la terneza sostenida y la jugosidad, a la vez que aumentó la percepción de tejido conectivo (cuadro 3). Con respecto al TM, se comprobó un aumento en la terneza inicial, terneza sostenida y jugosidad y un descenso en la percepción de tejido conectivo a los 15 d de maduración. El TV solo afectó la variable olor, la cual mostró valores más altos en el TV más corto (cuadro 3).

Cuadro 1. Hematocrito, proteínas plasmáticas totales, glucógeno muscular, glucemia, lactato plasmático y concentraciones de adrenalina y cortisol urinario en novillos sometidos a dos tiempos de viaje a la planta faenadora (TV) y dos tiempos de espera pre-faena (TE).

Haematocrit, total plasma protein, muscle glycogen, glycemia, plasmatic lactate and urinary concentration of adrenaline and cortisol in steers transported for $3 \mathrm{~h}$ or $8 \mathrm{~h}$ and with a lairage time of $2 \mathrm{~h}$ or $14 \mathrm{~h}$.

\begin{tabular}{|c|c|c|c|c|c|c|c|}
\hline & \multicolumn{2}{|c|}{ TV (h) } & \multicolumn{2}{|c|}{$\mathrm{TE}(\mathrm{h})$} & \multirow{2}{*}{$\mathrm{EE}$} & \multicolumn{2}{|c|}{$\mathrm{P}$} \\
\hline & 3 & 8 & 2 & 14 & & $\mathrm{TV}$ & TE \\
\hline Hematocrito (\%) & 41,6 & 42,2 & $40,7 \mathrm{a}$ & $43,0 \mathrm{~b}$ & 0,65 & NS & 0,01 \\
\hline PPT (g/100 ml) & 8,20 & 8,30 & $7,79 \mathrm{a}$ & $8,70 \mathrm{~b}$ & 0,156 & NS & $<0,01$ \\
\hline Glucógeno ( $\mu \mathrm{mol} / \mathrm{g}$ tej. hum.) & 33,40 & 38,50 & 33,10 & 38,80 & 2.350 & NS & NS \\
\hline Glucemia (mg/dl) & 7,10 & 7,57 & $7,82 b$ & $6,85 a$ & 0,303 & NS & 0,03 \\
\hline Lactato plasmático (mg/dl) & 42,01 & 44,75 & 45,12 & 41,64 & 2.494 & NS & NS \\
\hline Adrenalina urin. (ng/mg creat.) & 4,72 & 9,58 & $10,71 b$ & $3,59 \mathrm{a}$ & 2.294 & NS & 0,03 \\
\hline Cortisol urinario (ng/mg creat.) & 151 & 130 & $109 \mathrm{a}$ & $172 b$ & 13,1 & NS & $<0,01$ \\
\hline
\end{tabular}

EE: Error estándar de la media; PPT: proteínas plasmáticas totales; NS: no significativo; P < 0,05 indica significancia estadística; letras diferentes en la misma fila indican diferencias estadísticas entre medias en Test de Tukey $(\mathrm{P}<0,05)$.

EE: standard error of the mean; PPT: total plasma protein; NS: non significant; differences with $\mathrm{P}<0.05$ are statistically significant; means in the same file followed by different letter differ significantly $\mathrm{P}<0.05$. 
Cuadro 2. Resultados de los análisis físicos y de perfil de textura de la carne correspondiente al músculo Longissimus dorsi de novillos sometidos a dos tiempos de viaje a la planta faenadora (TV) y dos tiempos de espera pre-faena (TE), luego de cuatro tiempos de maduración (TM).

Physical and texture profile analysis results of Longissimus dorsi from steers transported for $3 \mathrm{~h}$ or $8 \mathrm{~h}$ and with a lairage time of $2 \mathrm{~h}$ or 14 $h$, after four different ageing times.

\begin{tabular}{|c|c|c|c|c|c|c|c|c|c|c|}
\hline & \multicolumn{2}{|c|}{$\mathrm{TV}(\mathrm{h})$} & \multicolumn{2}{|c|}{$\mathrm{TE}(\mathrm{h})$} & \multirow{2}{*}{$\mathrm{EE}$} & \multicolumn{4}{|c|}{$\mathrm{TM}(\mathrm{d})$} & \multirow{2}{*}{$\mathrm{EE}$} \\
\hline & 3 & 8 & 2 & 14 & & 4 & 6 & 8 & 15 & \\
\hline $\mathrm{pH} 24$ & 5,52 & 5,50 & 5,53 & 5,50 & 0,014 & - & - & - & - & - \\
\hline \multicolumn{11}{|l|}{ Color músculo } \\
\hline $\mathrm{L}^{*}$ & 33,33 & 33,97 & 33,52 & 33,77 & 0,579 & 34,25 & 32,65 & 33,43 & 34,26 & 0,718 \\
\hline$a^{*}$ & 12,79 & 12,91 & 12,64 & 13,05 & 0,221 & $12,15 b$ & $13,39 a$ & $12,50 \mathrm{~b}$ & $13,35 \mathrm{a}$ & 0,299 \\
\hline$b^{*}$ & 12,11 & 12,67 & 12,16 & 12,62 & 0,246 & 12,45 & 12,43 & 11,99 & 12,68 & 0,293 \\
\hline $\mathrm{WB}(\mathrm{N})$ & 34,04 & 33,31 & 32,71 & 34,63 & 0,979 & $34,32 \mathrm{a}$ & $33,88 \mathrm{a}$ & $36,40 \mathrm{a}$ & $30,10 b$ & 1.301 \\
\hline Mermas (\%) & 32,32 & 32,24 & 32,26 & 32,31 & 0,270 & $31,81 b$ & $32,99 a$ & $31,81 \mathrm{~b}$ & $32,52 \mathrm{ab}$ & 0,340 \\
\hline CRA $(\%)$ & 31,77 & 31,84 & 32,26 & 31,34 & 0,395 & 31,96 & 31,40 & 31,21 & 32,64 & 0,558 \\
\hline \multicolumn{11}{|l|}{ APT } \\
\hline Dureza $1(\mathrm{~N})$ & 88,29 & 88,23 & 86,92 & 89,60 & 1.591 & $92,30 \mathrm{a}$ & $87,58 \mathrm{ab}$ & $89,85 \mathrm{a}$ & $83,32 b$ & 2.161 \\
\hline Dureza $2(\mathrm{~N})$ & 76,49 & 76,44 & 75,18 & 77,74 & 1.447 & $80,00 \mathrm{a}$ & $76,13 \mathrm{ab}$ & $77,80 \mathrm{a}$ & $71,91 \mathrm{~b}$ & 1.940 \\
\hline Elasticidad & 0,49 & 0,49 & $0,48 \mathrm{a}$ & $0,50 \mathrm{a}$ & 0,004 & $0,49 \mathrm{a}$ & $0,48 \mathrm{a}$ & $0,49 a$ & $0,51 b$ & 0,006 \\
\hline Cohesividad & 0,51 & 0,51 & 0,50 & 0,51 & 0,003 & 0,50 & 0,51 & 0,51 & 0,50 & 0,004 \\
\hline Masticabilidad & 22,28 & 21,89 & $21,29 \mathrm{a}$ & $22,88 \mathrm{~b}$ & 0,520 & 22,71 & 21,50 & 22,40 & 21,73 & 0,735 \\
\hline
\end{tabular}

EE: Error estándar de la media; WB: fuerza de corte en cizalla de Warner-Brätzler; Mermas: mermas por cocción; CRA: capacidad de retención de agua; letras diferentes en la misma fila indican diferencias estadísticas entre medias en Test de Tukey $(\mathrm{P}<0,05)$.

EE: standard error of the mean; WB: Warner-Brätzler shear force; Mermas: cooking losses; CRA: water-holding capacity; means in the same file followed by different letter differ significantly $(\mathrm{P}<0.05)$.

Cuadro 3. Análisis de panel sensorial del músculo Longissimus dorsi de novillos sometidos a dos tiempos de viaje a la planta faenadora (TV) y dos tiempos de espera pre-faena (TE), luego de cuatro tiempos de maduración (TM).

Sensory analysis of Longissimus dorsi from steers transported for $3 \mathrm{~h}$ or $8 \mathrm{~h}$ and with a lairage time of $2 \mathrm{~h}$ or $14 \mathrm{~h}$, after four different ageing times.

\begin{tabular}{|c|c|c|c|c|c|c|c|c|c|c|}
\hline & \multicolumn{2}{|c|}{ TV (h) } & \multicolumn{2}{|c|}{ TE (h) } & \multirow{2}{*}{$\mathrm{EE}$} & \multicolumn{4}{|c|}{$\mathrm{TM}(\mathrm{d})$} & \multirow{2}{*}{$\mathrm{EE}$} \\
\hline & 3 & 8 & 2 & 14 & & 4 & 6 & 8 & 15 & \\
\hline Olor & $6,03 a$ & $5,84 \mathrm{~b}$ & 5,94 & 5,91 & 0,070 & 5,89 & 6,01 & 5,99 & 5,81 & 0,100 \\
\hline Flavor & 6,12 & 6,11 & 6,14 & 6,08 & 0,060 & 6,15 & 6,16 & 5,97 & 6,18 & 0,100 \\
\hline Terneza inicial & 5,98 & 5,96 & $6,17 \mathrm{a}$ & $5,77 \mathrm{~b}$ & 0,070 & $5,87 \mathrm{~b}$ & $5,87 \mathrm{~b}$ & $5,79 b$ & $6,37 \mathrm{a}$ & 0,140 \\
\hline Terneza sost. & 6,64 & 6,63 & $6,81 \mathrm{a}$ & $6,46 b$ & 0,110 & $6,56 \mathrm{ab}$ & $6,48 b$ & $6,45 b$ & $7,06 \mathrm{a}$ & 0,140 \\
\hline Jugosidad & 5,94 & 6,04 & $6,09 a$ & $5,88 \mathrm{~b}$ & 0,090 & $5,88 \mathrm{~b}$ & $5,90 \mathrm{~b}$ & $5,92 b$ & $6,26 \mathrm{a}$ & 0,110 \\
\hline Tej. Conectivo & 3,95 & 3,98 & $3,83 \mathrm{~b}$ & $4,10 \mathrm{a}$ & 0,080 & $4,14 \mathrm{a}$ & $4,01 \mathrm{a}$ & $4,23 \mathrm{a}$ & $3,48 b$ & 0,100 \\
\hline
\end{tabular}

EE: Error estándar de la media; letras diferentes en la misma fila indican diferencias estadísticas entre medias en Test de Tukey $(\mathrm{P}<0,05)$.

EE: standard error of the mean; means in the same file followed by different letter differ significantly $(\mathrm{P}<0.05)$.

\section{DISCUSIÓN}

Para facilitar la lectura de la discusión y debido a que no hubo interacciones significativas, la misma se hace analizando en forma separada cada uno de los factores (TV, TE y TM). A modo de síntesis, se discute el escaso o nulo efecto del TV sobre las variables fisiológicas y de calidad de carne, el efecto significativo del TE tanto sobre variables fisiológicas como sobre la calidad sensorial de la carne y, por último, los efectos más notorios del TM tanto sobre la calidad instrumental como sensorial de la carne. 


\section{TIEMPO DE VIAJE}

El TV no afectó ninguna de las variables fisiológicas analizadas. Los resultados muestran que $3 \mathrm{~h}$ vs $8 \mathrm{~h}$ de TV no modificaron el status de hidratación de los animales, al menos no lo suficiente como para modificar significativamente el hematocrito y las proteínas plasmáticas totales. Tampoco los glúcidos ni las hormonas de estrés fueron afectados, por lo que debe suponerse que la combinación entre intensidad y duración del estímulo estresante no fue suficiente como para producir alteraciones duraderas que se reflejaran al momento de la faena. Varios estudios han demostrado que a medida que avanza el viaje los animales logran cierto nivel de adaptación al ambiente estresante del camión y que el pico en la concentración plasmática cortisol se produce en las primeras horas de viaje para luego dar lugar a un descenso gradual (Warriss y col 1995, Parker y col 2009). Esto se comprueba también cuando se miden variables fisiológicas como la frecuencia cardíaca (Eldrige y col 1988) o la temperatura rectal (Pettiford y col 2008). Sin embargo, esta adaptación no contradice el hecho de que viajes más largos implican un mayor período de deprivación de agua y alimentos y una mayor fatiga acumulada (Fisher y col 2009).

En forma coherente con los resultados fisiológicos, tampoco se detectaron efectos importantes del TV sobre la calidad de carne, tanto en el análisis instrumental como en el sensorial. Gallo y col (2000), trabajando sobre LD de animales transportados en otoño-invierno, tampoco encontraron efecto en $\mathrm{pH}$ final ni en luminosidad (parámetro $\mathrm{L}^{*}$ ) cuando evaluaron viajes de hasta $12 \mathrm{~h}$. Sin embargo, dichos autores sí encontraron un descenso significativo en los parámetros $\mathrm{a}^{*} \mathrm{y}$ b* con viajes de $6 \mathrm{~h}$. Batista de Deus y col (1999) encontraron efectos significativos en pH final en viajes más cortos que el practicado en nuestro ensayo, aunque se trataba de animales terminados en pastoreo sin suplementación con fuentes almidonosas. Maria y col (2003) encontraron leves efectos de viajes hasta 6 $\mathrm{h}$ en variables instrumentales de calidad de carne como WB o color cuando los animales utilizados procedían de un engorde a corral basado en concentrados. Villarroel y col (2003) no encontraron efecto de tiempo de viaje sobre variables evaluadas por un panel sensorial. Según María (2008), el tiempo de viaje sería la variable principal que determina el costo biológico del viaje. Sin embargo, Fisher y col (2009) consideran que las condiciones en las que se desarrolla el viaje es el determinante principal del efecto estresante, más allá del tiempo de viaje. Otras variables, como la calidad de manejo, la condición nutricional previa de los animales (Immonen y col, 2000a $2000^{\mathrm{b}}$ ) y calidad de rutas hacen que en algunos casos no resulte fácil comparar distancias o tiempos de viaje diferentes y sus efectos sobre el nivel de estrés y la calidad de carne de los animales. Bajo las condiciones de nuestro estudio, los resultados parecen indicar que 8 horas de viaje no significaron un estímulo suficiente en intensidad y/o duración como para producir efectos detectables sobre la calidad de la carne. Lamentablemente, no se registró la información sobre temperatura y humedad durante el viaje, lo cual podría haber contribuido a una interpretación más profunda de los resultados.

\section{TIEMPO DE ESPERA}

Dentro de las variables fisiológicas, el TE afectó el hematocrito, las proteínas plasmáticas totales, la glucemia y la concentración urinaria de hormonas de estrés. El hematocrito puede aumentar tanto producto de la deshidratación (Tadich y col 2005) como por liberación de eritrocitos al torrente sanguíneo desde el bazo producto de la adrenalina liberada (Tarrant y col 1992, Warriss y col 1995, Gupta y col 2007). El hecho de que las proteínas plasmáticas totales también estuvieran elevadas en el TE de 14 h, hace pensar que se trató de un proceso de deshidratación (Knowles y col 1999). Adicionalmente, lo niveles más altos de cortisol urinario podrían haber aumentado la diuresis en el TE de $14 \mathrm{~h}$, aumentando el cuadro de deshidratación (Parker y col 2003). A pesar de contar con agua disponible en los corrales de espera, los animales no lograron reponer las pérdidas de líquidos y se mostraron más deshidratados, lo cual coincide con los hallazgos de Jarvis y col (1996). Dado que no se realizó un estudio observacional del comportamiento durante la espera, solo se puede especular acerca de las posibles causas. Una de las posibles explicaciones sería la que los bebederos fuesen demasiado pequeños como para posibilitar que todos los animales bebieran y que por un efecto de dominancia social y competencia por los recursos, solo una parte de los animales lograse hidratarse correctamente (Petherick 2007, Petherick y Phillips 2009). Otra causa posible sería la temperatura del agua, la cual podría haber estados muy fría o muy caliente, conduciendo a que los animales no beban. Otros autores (Tadich y col 2005, Knowles y col 1999, Jarvis y col 1996) mencionan que el ambiente novedoso de los corrales podría afectar el comportamiento normal de los animales e impedir que los animales beban normalmente.

La menor glucemia en el TE de $14 \mathrm{~h}$, podría explicarse tanto por el ayuno más prolongado como por los descensos registrados en la concentración de adrenalina, hormona fuertemente hiperglucemiante. Además, el descenso en los niveles de adrenalina permitiría actuar a la insulina, reduciendo los niveles plasmáticos de glucosa (Chiasson y col 1981). Knowles y col (1999) también encontraron un descenso de la glucemia en bovinos luego de 12 h de recuperación post-transporte, a pesar de que los niveles de cortisol se mantuvieron altos.

Los valores promedio de glucógeno muscular registrados (36 $\mu \mathrm{mol} / \mathrm{g}$ tej hum) son lo suficientemente altos para permitir alcanzar bajos $\mathrm{pH}$ finales en la carne. Esto coincide con los hallazgos de Lowe y col (2004), quienes encontraron que concentraciones de glucógeno muscular 
de $25 \mu \mathrm{mol} / \mathrm{g}$ tej hum medidos a los $45 \mathrm{~min}$ post sacrificio serían suficientes para alcanzar un bajo $\mathrm{pH}$ final.

Los resultados encontrados en adrenalina y cortisol urinarios son coherentes con el hecho de que ambas hormonas poseen una vida media diferente y representan etapas diferentes en la respuesta fisiológica al estrés. Mientras que la adrenalina es una hormona de vida media corta, cuyos niveles aumentan y descienden rápidamente, el cortisol es una hormona de vida media prolongada, que aumenta gradualmente y se mantiene alta mientras persiste el efecto estresante (Matteri y col 2000). Los animales que tuvieron un TE prolongado mostraron un descenso en la adrenalina urinaria y un aumento en el cortisol urinario. En forma opuesta, los animales con TE breve mostraron mayores niveles de adrenalina urinaria, posiblemente reflejando el estatus de los momentos finales del viaje y los momentos iniciales de la llegada a la planta faenadora. El aumento en cortisol con el TE más prolongado coincide con los hallazgos de Tadich y col (2005).

Los efectos del TE sobre la calidad de carne fueron más notorios, específicamente afectando el perfil de textura y los resultados del panel sensorial. En líneas generales, el efecto de mayor TE fue negativo sobre la calidad de la carne, lo cual coincide con los hallazgos de Gallo y col (2003). El TE de $14 \mathrm{~h}$ produjo aumentos en la masticabilidad y la elasticidad (cuadro 2). Un aumento en la masticabilidad indica mayor trabajo para reducir un alimento para que pueda ser deglutido e implica mayor tiempo de masticación de la muestra (Szczesniak 2002) y es un producto de la dureza, la cohesividad y la elasticidad. Por su parte, la elasticidad refleja la tasa a la cual un alimento recupera la forma original luego de ser deformado (Szczesniak 2002). Ambos aumentos estarían indicando una disminución en la calidad sensorial de la carne, dato que se verifica en el panel sensorial, donde los valores de terneza inicial, terneza sostenida y jugosidad descienden y la percepción de tejido conectivo aumenta con un mayor TE (cuadro 3). Ruiz de Huidobro y col (2005) encontraron que la masticabilidad tiene relación negativa con terneza y jugosidad evaluadas por panel sensorial. También Caine y col (2003) encontraron correlaciones negativas entre masticabilidad y terneza, y positivas entre masticabilidad y percepción de tejido conectivo en el panel sensorial.

\section{TIEMPO DE MADURACIÓN}

En el análisis instrumental, el TM tuvo efectos estadísticamente significativos tanto en WB como en dureza 1 y dureza 2 en el perfil de textura (cuadro 2). También se verifican cambios similares en el panel sensorial, donde la terneza y la jugosidad aumentan a los $15 \mathrm{~d}$ de TM (cuadro 3).

El valor promedio de WB medido fue de $33,67 \mathrm{~N}$, valor que se considera propio de muestras muy tiernas (Shackelford y col 1991). Los valores son menores que los en- contrados en la bibliografía por Sullivan y Calkins (2011) para el mismo músculo, aunque son cercanos a los encontrados por Belew y col (2003). Estos bajos valores de WB posiblemente se deban a que se trató de animales jóvenes (menos de 30 meses) y de raza Angus, características que han sido relacionadas por otros autores con bajos valores de WB y alta terneza (Shorthose y Harris 1990, Franco y col 2008). Es importante resaltar que aun los tratamientos sometidos a TM más breves mostraron altos niveles de terneza y valores de WB bajos, lo cual indicaría que aun con poco TM este tipo de animales produciría carne tierna.

El efecto del TM sobre el color fue leve e irregular, afectando únicamente al parámetro a*. Papaleo Mazzucco y col (2010) reportaron un aumento de los parámetros L*, $a^{*}$ y b* con tiempos de maduración de 14 d, lo cual coincide también con lo reportado por Boakye y Mittal (1996). Sin embargo, Oliete y col (2006) encontraron aumentos en los parámetros $\mathrm{a}^{*} \mathrm{y} \mathrm{b}^{*}$, sin que se encuentre afectado el parámetro L*. Alende y col (2012) encontraron aumentos en los parámetros $\mathrm{a}^{*} \mathrm{y} \mathrm{b}^{*}$, pero un comportamiento irregular en el parámetro $\mathrm{L}^{*}$. Aumentos en el parámetro $\mathrm{a}^{*}$ serían esperables con mayores TM como producto de una menor actividad de las enzimas oxígeno-dependiente a lo largo de la maduración, lo que aumentaría el estado de oxigenación de la mioglobina y daría un color más rojizo (Boakye y Mittal 1996). Por otro lado, un aumento en el parámetro b* sería esperable por una mayor concentración de metamioglobina con mayor TM, debido a un cambio en el estado de oxidación del hierro en la molécula de mioglobina (Oliete y col 2006; Papaleo Mazzucco y col 2010). Sin embargo, Ruiz de Huidobro y col (2003) tampoco encontraron un patrón regular y claro en la variación del color a lo largo del TM, e incluso observaron respuestas diferentes según la carne procediera de vaquillonas o de toros. Esta irregularidad en el comportamiento del color con la maduración podría deberse al hecho de que se trató de muestras envasadas al vacío, que según algunos autores podrían tener más variabilidad en color como respuesta a la maduración (Eikelenbom y col 2000).

Se concluye que, bajo las condiciones de nuestro estudio, un tiempo de viaje de hasta $8 \mathrm{~h}$ no tuvo efectos negativos sobre los indicadores de estrés o la calidad de carne. Aun cuando el tiempo de viaje debe ser considerado una variable importante que afecta el bienestar animal, las condiciones en las que se realiza ese viaje podrían ser más importantes que la duración del viaje en sí mismo. La industria cárnica afronta hoy un escenario en el cual probablemente los tiempos de viaje aumenten, por lo cual es importante tomar decisiones que optimicen la eficiencia de la cadena logística, lo cual redundaría en beneficios tanto desde el punto de vista del bienestar animal y calidad de producto como desde un enfoque económico (Miranda de la Lama y col 2013) y ambiental. Una gestión eficiente de dicha cadena en la cual se coordinen todos los eslabones de la cadena logística debería ser un objetivo para la industria cárnica. 
Por otro lado, tiempos de espera más largos se reflejaron en un aumento en los indicadores fisiológicos de estrés y afectaron negativamente ciertas variables de calidad de la carne. Aquí cabe recalcar que las condiciones de espera son probablemente tan importantes como el tiempo de espera, por lo cual, en particular en América Latina, los esfuerzos por mejorar las condiciones de espera en corrales previo a la faena será beneficioso para el bienestar animal y la calidad del producto. Surge como aspecto importante la disponibilidad de espacio para descanso y bebida de manera que se reduzca el impacto de la competencia y la escala de dominancia permitiendo alcanzar una correcta recuperación de reservas energéticas y una buena hidratación en los animales.

En términos generales, tiempos de maduración de 15 $\mathrm{d}$ mejoraron levemente la terneza de la carne, aunque tanto los parámetros instrumentales como sensoriales de las carnes fueron compatibles con carnes de alta calidad aun en los tiempos de maduración más breves. La industria cárnica deberá profundizar sus investigaciones para establecer el uso más eficiente de recursos que conduzcan a la mejor calidad de producto teniendo en cuenta el tipo, edad y condición del animal, ya que parece difícil concluir que exista un tiempo de maduración óptimo aplicable a todos los casos.

\section{REFERENCIAS}

Alende M, G Volpi Lagreca, G Grigioni, AJ Pordomingo, D Pighín, F Carduza, F Babinec. 2012. Efecto de la utilización de perros durante el arreo y de la maduración sobre parámetros físicos de carne bovina. Rev Arg Prod Anim 32, 165-173.

AMSA, American Meat Science Association. 1996. Meat evaluation handbook, Chicago, IL, USA.

Batista de Deus JC, WP Silva, GJD Soares. 1999. Efeito da distância de transporte de bovinos no metabolismo postmortem. Rev Bras Agroc 5, 152-156.

Belew JB, JC Brooks, DR McKenna, JC Savell. 2003. WarnerBratzler shear evaluations of 40 bovine muscles. Meat Sci 64, 507-512.

Boakye K, GS Mittal. 1996. Changes in colour of beef Longissimus dorsi muscle during ageing. Meat Sci 42, 347-354.

Bradford MM. 1976. A rapid and sensitive method for the quantitation of microgram quantities of protein utilizing the principle of protein-dye binding. Anal Biochem 72, 248-254.

Broom DM. 2008. The welfare of livestock during road transport. In: Appleby MC, Cussen VA, Garcés L, Lambert IA, Turner J (eds). Long distance transport and welfare offarm animals. CAB International, Oxfordshire, UK, Pp 157-181.

Bruas-Reignier F, J Brun-Bellut. 1996. Changes affecting the Longissimus dorsi, Triceps brachii caput longum and Rectus femoris muscles of young friesian bulls during meat ageing. Meat Sci 43, 335-344.

Caine WR, JL Aalhus, DR Best, MER Dugan, LE Jeremiah. 2003. Relationship of texture profile analysis and Warner-
Bratzler shear force with sensory characteristics of beef rib steaks. Meat Sci 64, 333-339.

Campo MM, C Sañudo, B Panea, P Alberti, P Santolaria. 1999. Breed type and ageing time effects on sensory characteristics of beef strip loin steaks. Meat Sci 51, 383-390.

Casagrande G, ME Deanna, MA Farrell, F J Babinec. 2012. Estadísticas agroclimáticas de la EEA Anguil "Ing. Agr. Guillermo Covas”. Periodo 1973-2011. Ediciones INTA, Anguil, La Pampa, Argentina.

Chiasson JL, H Shikama, DTW Chu, JH Exton. 1981. Inhibitory effect of epinephrine on insulin-stimulated glucose uptake by rat skeletal muscle. J Clin Invest 68, 706-713.

Eikelenboom G, AH Hoving-Bolink, I Kluitman, JH Houben, RE Klont. 2000. Effect of dietary vitamin E supplementation on beef colour stability. Meat Sci 54, 17-22.

Eldridge GA, CG Winfield, DJ Cahill. 1988. Responses of cattle to different space allowances, pen sizes and road conditions during transport. Australian J Exp Agr 28, 155-159.

Ferguson DM, RD Warner. 2008. Have we underestimated the impact of pre-slaughter stress on meat quality in ruminants? Meat Sci 80, 12-19.

Fisher AD, IG Colditz, C Lee, DM Ferguson. 2009. The influence of land transport on animal welfare in extensive farming systems. $J$ Vet Behav 4, 157-162.

Franco J, O Feed,G Garibotto, F Ballesteros, E Forichi, O Bentancur, G Bianchi. 2008. Efecto de la maduración sobre la textura y calidad sensorial de la carne de vaquillonas Hereford. Rev Arg Prod Anim 28, 39-44.

Gallo C, S Perez, C Sanhueza, J Gasic. 2000. Efectos del tiempo de transporte de novillos previo al faenamiento sobre el comportamiento, las pérdidas de peso y algunas características de la canal. Arch Med Vet 32, 157-170.

Gallo C, MA Espinoza, J Gasic. 2001. Efectos del transporte por camión durante 36 horas con y sin período de descanso sobre el peso vivo y algunos aspectos de calidad de carne en bovinos. Arch Med Vet 33, 43-53.

Gallo C, G Lizondo, TG Knowles. 2003. Effects of journey and lairage time on steers transported to slaughter in Chile. Vet Rec 152, 361-364.

Gallo C, P Warriss, T Knowles, R Negrón, A Valdés, I Mencarini. 2005. Densidades de carga utilizadas para el transporte de bovinos destinados a matadero en Chile. Arch Med Vet 37, 155-159.

Grandin T. 1997. Assessment of stress during handling and transport. J Anim Sci 75, 249-257.

Gupta S, B Earley, MA Crowe. 2007. Effect of 12-hour road transportation on physiological, immunological and haematological parameters in bulls housed at different space allowances. Vet $J$ 173, 605-616.

Herrera-Mendez CH, S Becilab, A Boudjellalb, A Ouali. 2006. Meat ageing: reconsideration of the current concept. Trends Food Sci Tech 17, 394-405.

Honkavaara, M, E Helynranta, J Ylönen, T Pudas. 2005. Effect of chassis vibration during road transport on cattle welfare and meat quality. Proc. of the 51st congress of meat science and technology, Baltimore, USA, Pp 1-5.

Huertas-Canén SM. 2009. Buenas prácticas de manejo durante el embarque y transporte a la planta de sacrificio. En: D Mota-Rojas D, Guerrero-Legarreta I, Trujillo-Ortega ME (eds). Bienestar Animal y Calidad de la Carne. México DF, México, Pp 73-84. 
Immonen K, M Ruusunen, K Hissa, E Puolanne. 2000a. Bovine muscle glycogen concentration in relation to finishing diet, slaughter and ultimate pH. Meat Sci 55, 25-31.

Immonen K, DM Schaefer, E Puolanne, RG Kauman, EV Nordheim. $2000^{\mathrm{b}}$. The relative effect of dietary energy density on repleted and resting muscle glycogen concentrations. Meat Sci 55, 155-162.

Jarvis AM, DWJ Harrington, MS Cockram. 1996. Effect of source and lairage on some behavioural and biochemical measurements of feed restriction and dehydration in cattle at a slaughterhouse. Appl Anim Behav Sci 50, 83-94.

Knowles TG, PD Warriss, SN Brown, JE Edwards. 1999. Effects on cattle of transportation by road for up to 31 hours. Vet Rec 145, 575-582.

Lowe, TE, CE Devine, RW Wells, LL Lynch. 2004. The relationship between post-mortem urinary catecholamines, meat ultimate $\mathrm{pH}$, and shear force in bulls and cows. Meat Sci 67, 251-260.

María GA, M Villarroel, C Sañudo, JL Olleta, G Gebresenbet. 2003. Effect of transport time and ageing on aspects of beef quality. Meat Sci 65, 1335-1340.

María GA. 2008. Meat quality. In: Appleby MC, Cussen VA, Garcés L, Lambert IA, Turner J (eds). Long distance transport and welfare of farm animals. CAB International, Oxfordshire, UK, Pp 77-112.

Matteri RL, JA Carroll, CJ Dyer. 2000. Neuroendocrine responses to stress. In: Moberg G, Mench JA (eds). The biology of animal stress. CAB International, Wallingford, UK, Pp 43-76.

Miranda de la Lama GC. 2013. Transporte y logística pre-sacrificio: principios y tendencias en bienestar animal y su relación con la calidad de la carne. Vet Mexico 44, 31-56.

Miranda de la Lama GC, M Villarroel, JL Olleta, S Alierta, C Sañudo, GA Maria. 2009. Effect of the pre-slaughter logistic chain on meat quality of lambs. Meat Sci 83, 604609.

Miranda de la Lama GC, M Pascual-Alonso, A Guerrero, P Alberti, S Alierta, P Sanz, JP Gajan, M Villarroel, A Dalmau, A Velarde, MM Campo, F Galindo, MP Santolaria, C Sañudo, GA María. 2013. Influence of social dominance on production, welfare and the quality of meat from beef bulls. Meat Sci 94, 432-437.

Mounier L, I Veissier, S Andanson, E Delval, A Boissy. 2006. Mixing at the beginning of fattening moderates social buffering in beef bulls. Appl Anim Behav Sci 96, 185-200.

Oliete B, JA Carballo, A Varela, T Moreno, L Monserrat, L Sánchez. 2006. Effect of weaning status and storage time under vacuum upon physical caracteristics of meat of the Rubia Gallega breed. Meat Sci 73, 102-108.

Papaleo Mazzucco J, LM Melucci, EL Villarreal, CA Mezzadra, L Soria, P Corva, MM Motter, A Schor, MC Miquel. 2010. Effect of ageing and $\mu$-calpain markers on meat quality from Brangus steers finished on pasture. Meat Sci 86, 878-882.

Parker AJ, GP Hamlin, CJ Coleman, LA Fitzpatrick. 2003. Dehydration in stressed ruminants may be the result of a cortisol-induced diuresis. J Anim Sci 81, 512-519.

Parker AJ, CJ Coleman, LA Fitzpatrick. 2009. A technique for sampling blood from cattle during transportation. Anim Prod Sci 49, 1068-1070.

Parrott RF, SJG Hall, DM Lloyd. 1998. Heart rate and stress hormone responses of sheep to road transport following two different loading responses. Anim Welfare 7, 257-267.

Passonneau J, V Lauderdale. 1974. A comparison of three methods of glycogen measurement in tissues. Anal Biochem 60, 405-412.

Petherick JC, 2007. Spatial requirements of animals: Allometry and beyond. Appl Anim Behav Sci 2, 197-204.

Petherick JC, CJC Phillips. 2009. Space allowances for confined livestock and their determination from allometric principles. Appl Anim Behav Sci 117, 1-12.

Pettiford SG, DM Ferguson, JM Lea, C Lee, DR Paull, MT Reed, GN Hinch, AD Fisher. 2008. The effect of loading practices and 6 hour road transport on the physiological responses of yearling cattle. Aust J Exp Agric 48, 10281033.

Romero MH, LF Uribe-Velasquez, JA Sánchez, GC Miranda de la Lama. 2013. Risk factors influencing bruising and high muscle $\mathrm{pH}$ in Colombian cattle carcasses due to transport and pre-slaughter operations. Meat Sci 95, 256-263.

Ruiz de Huidobro F, E Miguel, B Blázquez. 2003. Changes in meat quality characteristics of bovine meat during the first 6 days post mortem. Meat Sci 65, 1439-1446.

Ruiz de Huidobro F, E Miguel, B Blázquez, E Onega. 2005. A comparison between two methods (Warner-Bratzler and texture profile analysis) for testing either raw meat or cooked meat. Meat Sci 69, 527-536.

SAS. 1990. SAS User's Guide: Statistics. SAS Inst., Inc., Cary, Nc.

Shackelford SD, JB Morgan, HR Cross, JW Savell. 1991. Identification of threshold levels for warner-bratzler shear force in beef top loin steaks. J Muscle Foods 2, 289-296.

Shorthose WR, PV Harris. 1990. Effect of animal age on the tenderness of selected beef muscles. J Food Sci 55, 1-8.

Sullivan GA, CR Calkins. 2011. Ranking beef muscles for Warner Bratzler shear force and trained sensory panel ratings from published literature. J Food Quality 34, 195-203.

Szczesniak AS. 2002. Texture is a sensory property. Food Qual Prefer 13, 215-225.

Tadich N, C Gallo, M Alvarado. 2000. Efectos de 36 horas de transporte terrestre con y sin descanso sobre algunas variables sanguíneas indicadoras de estrés en bovinos. Arch Med Vet 32, 171-183.

Tadich N, C Gallo, R Echeverría, G Van Schaik. 2003. Efecto del ayuno durante dos tiempos de confinamiento y transporte terrestre sobre algunas variables sanguíneas indicadoras de estrés en novillos. Arch Med Vet 35, 171-185.

Tadich N, C Gallo, H Bustamante, M Schwerter, G van Schaik. 2005. Effect of transport and lairage time on some blood constituents of Fresian-cross steers in Chile. Livest Prod Sci 93, 223-233.

Tarrant PV, FJ Kenny, D Harrington. 1988. The effect of stocking density during 4 hour transport to slaughter on behaviour, blood constituents and carcass bruising in Fresian steers. Meat Sci 24, 209-222.

Tarrant PV, FJ Kenny, D Harrington, M Murphy. 1992. Long distance transportation of steers to slaughter: effect of stocking density on physiology, behavior and carcass quality. Livest Prod Sci 30, 223-238.

Tarrant PV, T Grandin. 2000. Cattle transport. In: Grandin T (ed). Livestock handling and transport. CAB International, Oxfordhire, UK, Pp 151-174. 
Trinder P. 1969. Determination of glucose in blood using glucose oxidase with an alternative oxygen acceptor. Ann Clin Biochem 6, 24-27.

Villarroel M, GA María, C Sañudo, JL Olleta, G Gebresenbet. 2003. Effect of transport time on sensorial aspects of beef meat quality. Meat Sci 63, 353-357.
Warriss PD, SN Brown, TG Knowles, SC Kestin, JE Edwards, SK Dolan, AJ Phillips. 1995. Effects of cattle transport by road for up to 15 hours. Vet Rec 136, 319-323.

Watanabe A, CC Daly, CE Devine, 1996. The effects of the ultimate $\mathrm{pH}$ of meat on tenderness changes during ageing. Meat Sci 42, 67-78. 
